\title{
A note on the Taylor series expansions for multivariate characteristics of classical risk processes
}

\author{
M.A. Usábel ${ }^{\mathrm{a}, \mathrm{b}, *}$ \\ a Departamento Economia de la Empresa, Universidad Carlos III, Madrid, Spain \\ ${ }^{\mathrm{b}}$ Department of Actuarial Mathematics and Statistics, Heriot-Watt University, Edinburgh EH14 4AS, UK
}

\begin{abstract}
The series expansion introduced by Frey and Schmidt (1996) [Taylor Series expansion for multivariate characteristics of classical risk processes. Insurance: Mathematics and Economics 18, 1-12.] constitutes an original approach in approximating multivariate characteristics of classical ruin processes, specially ruin probabilities within finit time with certain surplus prior to ruin and severity of ruin. This approach can be considered alternative to inversion of Laplace transforms for particular claim size distributions [Gerber, H., Goovaerts, M., Kaas, R., 1987. On the probability and severity of ruin. ASTIN Bulletin 17(2), 151-163; Dufresne, F., Gerber, H., 1988a. The probability and severity of ruin for combinations of exponential claim amount distributions and their translations. Insurance: Mathematics and Economics 7, 75-80; Dufresne, F., Gerber, H., 1988b. The surpluses immediately before and at ruin, and the amount of the claim causing ruin. Insurance: Mathematics and Economics 7 , 193-199.] or discretization of the claim size and time [Dickson, C., 1989. Recursive calculation of the probability and severity of ruin. Insurance: Mathematics and Economics 8, 145-148; Dickson, C., Waters, H., 1992. The probability and severity of ruin in finit and infinit time. ASTIN Bulletin 22(2), 177-190; Dickson, C., 1993. On the distribution of the claim causing ruin. Insurance: Mathematics and Economics 12, 143-154.] applying the so-called Panjer's recursive algorithm [Panjer, H.H., 1981. Recursive calculation of a family of compound distributions. ASTIN Bulletin 12, 22-26.].

We will prove that the recursive relation involved in the calculations of the the $n$th derivative with respect to $\lambda-$ average number of claims in the time unit - of the multivariate finit time ruin probability (developed in the original paper by Frey and Schmidt (1996) can be simplified The cited simplificatio leads to a substantial reduction in the number of multiple integrals used in the calculations and makes the series expansion approach more appealing for practical implementation.
\end{abstract}

Keywords: Finite time ruin probability; Surplus prior to ruin; Severity of ruin; Series expansion; Recursive methods

\section{Introduction}

Let us defin a classical risk process in continuous time $\left\{Z_{t}\right\}_{t \geq 0}$ with $U_{k}$ claim sizes and premium $c$ per time unit,

* Tel.: +34-91-619-8917; fax: +34-91-571-0114

E-mail address: ausabel@correo.cop.es (M.A. Usábel) 


$$
Z_{t}=u+c t-\sum_{k=1}^{N_{t}} U_{k}
$$

where $u$ are the initial reserves, and $N_{t}$ the total number of claims up to time $t$ (with d.f. of the waiting times between claims $w(t)$ ), where $\lambda$ is the average number of claims in one year. Let $B$ denote the distribution function of claim sizes $U_{k}$ with mean $\mu^{-1}$ and $c=\lambda \mu^{-1}(1+\theta)$, where $\theta$ is the premium loading factor.

Let us now introduce $\tau=\inf \left\{w>0: Z_{w}<0\right\}$ as the ruin time and $Y=-Z_{\tau}$ as the defici at ruin time or severity of ruin and $X=Z_{\tau^{-}}$as the surplus just before the ruin.

If we consider a time horizon of $t$ years (or time units), the finit time multivariate probability of ruin with initial reserves $u$, arrival intensity $\lambda$ and severity of ruin less than $y$ and surplus less than $x$, is define as

$$
P\{\tau<t, X \leq x, Y \leq y\}=\Psi_{t, u, x, y}(\lambda) .
$$

When the time horizon is infinite the multivariate ultimate ruin probability can be expressed as

$$
P\{\tau<\infty, X \leq x, Y \leq y\}=\Psi_{u, x, y}(\lambda) .
$$

Frey and Schmidt (1996) used the Taylor series expansion (1.2) for the probability of ruin with time span $t, \Psi_{t, u, x, y}$ when the classical case of risk theory is considered, $w(t)=\lambda \mathrm{e}^{-\lambda t}$, restricted to the case when the premium loading factor was defined

$$
\begin{aligned}
& \theta=\frac{1-\lambda \mu^{-1}}{\lambda \mu^{-1}}, \quad \theta>0 \\
& \Psi_{t, u, x, y}(\lambda)=\sum_{n=1}^{\infty} \frac{\Psi_{t, u, x, y}^{(n)}(0)}{n !} \lambda^{n}, \quad \lambda \geq 0
\end{aligned}
$$

they proved that the function is analytic at $\lambda=0$ and the Taylor series expansion (1.2) has an infinit radius of convergence. They also specifie a recursive formula to obtain the $n$th derivative at $\lambda=0$ (Theorem 2 of the original paper by Frey and Schmidt (1996)).

Theorem 1 (Frey and Schmidt, 1996). For each $n \geq 1,0 \leq u, t<\infty, 0<x, y \leq \infty$, it holds

$$
\frac{\Psi_{t, u, x, y}^{(n)}(0)}{n !}=\frac{\Psi_{u, x, y}^{(n)}(0)}{n !}-\sum_{k=1}^{n} q_{t, u, x, y}^{(n-k, k)},
$$

where the quantities $q_{t, u, x, y}^{(n-k, k)}$ are given recursively by

$$
q_{t, u, x, y}^{(n, k)}=\int_{0}^{t} \int_{0}^{u+s} q_{t-s, u+s-z, x, y}^{(n-1, k)} b(z) \mathrm{d} z \mathrm{~d} s-\int_{0}^{t} q_{t-s, u+s, x, y}^{(n-1, k)} \mathrm{d} s
$$

and

$$
q_{t, u, x, y}^{(0, k)}=\frac{\Psi_{u+t, x, y}^{(k)}(0)}{k !}=F_{x, y} * G^{(k-1)}(u+t) .
$$

They also proved (in Theorem 1 of the original paper) that

$$
\frac{\Psi_{z, x, y}^{(k)}(0)}{k !}=F_{x, y} * G^{(k-1)}(z)
$$


where $G(w)=\int_{0}^{w}(1-B(z)) \mathrm{d} z$ and

$$
F_{x, y}(w)=\int_{w}^{\max \{x, w\}}(B(z+y)-B(z)) \mathrm{d} z .
$$

The Taylor series expansion (1.2) can be considered as an original and alternative approach in approximating multivariate characteristics of classical ruin processes other than inversion of Laplace transforms for particular claim size distributions (Gerber et al., 1987; Dufresne and Gerber, 1988a,b) or discretization of the claim size and time (Dickson, 1989, 1993; Dickson and Waters, 1992) applying the so-called Panjer's recursive algorithm (Panjer, 1981)).

Using the result of Frey and Schmidt (1996), the terms of the Taylor series expansion (1.2) are built upon the functions $q_{t, u, x, y}^{(i, k)}$. For a fxed $k$, the former functions are define recursively Eq. (1.4) starting with the initial function:

$$
q_{t, u, x, y}^{(0, k)}=F_{x, y} * G^{(k-1)}(u+t)
$$

then, for $i=1$

$$
q_{t, u, x, y}^{(1, k)}=\mathcal{H}_{1}\left(q_{t, u, x, y}^{(0, k)}\right)-\mathcal{H}_{2}\left(q_{t, u, x, y}^{(0, k)}\right),
$$

where integral operators $\mathcal{H}_{1}$ and $\mathcal{H}_{2}$ are define in general,

$$
\mathcal{H}_{1}\left(q_{t, u, x, y}^{(i-1, k)}\right)=\int_{0}^{t} \int_{0}^{u+s} q_{t-s, u+s-z, x, y}^{(i-1, k)} b(z) \mathrm{d} z \mathrm{~d} s, \quad \mathcal{H}_{2}\left(q_{t, u, x, y}^{(i-1, k)}\right)=\int_{0}^{t} q_{t-s, u+s-z, x, y}^{(i-1, k)} \mathrm{d} s .
$$

It is clear that $\mathcal{H}_{1}\left(q_{t, u, x, y}^{(i, k)}\right)$ or $\mathcal{H}_{2}\left(q_{t, u, x, y}^{(i, k)}\right)$ are also functions with multiple arguments $t, u, x, y$ and the same can be said about successive nested uses of the former operators, for instance $\mathcal{H}_{1}\left(\mathcal{H}_{1}\left(q_{t, u, x, y}^{(0, k)}\right)\right.$. These arguments will be omitted for the sake of simplicity.

For $i=2$,

$$
\begin{aligned}
q_{t, u, x, y}^{(2, k)} & =\mathcal{H}_{1}\left(\mathcal{H}_{1}\left(q_{t, u, x, y}^{(0, k)}\right)-\mathcal{H}_{2}\left(q_{t, u, x, y}^{(0, k)}\right)\right)-\mathcal{H}_{2}\left(\mathcal{H}_{1}\left(q_{t, u, x, y}^{(0, k)}\right)-\mathcal{H}_{2}\left(q_{t, u, x, y}^{(0, k)}\right)\right) \\
& =\mathcal{H}_{1}\left(\mathcal{H}_{1}\left(q_{t, u, x, y}^{(0, k)}\right)\right)-\mathcal{H}_{1}\left(\mathcal{H}_{2}\left(q_{t, u, x, y}^{(0, k)}\right)\right)-\mathcal{H}_{2}\left(\mathcal{H}_{1}\left(q_{t, u, x, y}^{(0, k)}\right)\right)+\mathcal{H}_{2}\left(\mathcal{H}_{2}\left(q_{t, u, x, y}^{(0, k)}\right)\right) .
\end{aligned}
$$

It is not hard to prove that the number of multiple integrals with known integrands, $q_{t, u, x, y}^{(0, k)}$ and $b(z)$, to be solved in the formula for $q_{t, u, x, y}^{(j, k)}$ will be $2^{j}$. As a consequence, for the $i$ th term of the Taylor series expansion, $\Psi_{t, u, x, y}^{(i)}(0) / i$ !, the total number integrals is

$$
\sum_{j=1}^{i-1} 2^{j}=2^{i}-2
$$

(it can be easily proved using formula (1.3)).

Finally, in order to obtain the Taylor expansion of order $n$, the total number of integrals is (see (1.2))

$$
\sum_{i=1}^{n}\left(2^{i}-2\right)=2^{n+1}-(n+1) 2
$$

It would be particularly useful in order to increase the applicability aspects of this approach that this exponentially growing number of multiple integrals (see Table 1, second column) could be reduced.

In Section 2, we will show with Theorem 2 that the recursive scheme, based on formula (1.4), used in the original paper by Frey and Schmidt (1996) can be simplified The cited simplificatio enhances the implementation of the 


\begin{tabular}{lcc}
\hline$n$ & Theorem $1\left(2^{n+1}-(n+1) 2\right)$ & Theorem 2 $(n(n-1) / 2)$ \\
\hline 1 & 0 & 0 \\
2 & 2 & 1 \\
3 & 8 & 3 \\
4 & 22 & 6 \\
5 & 52 & 10 \\
6 & 114 & 15 \\
7 & 240 & 21 \\
8 & 494 & 28 \\
9 & 1004 & 36 \\
10 & 2026 & 45 \\
\hline
\end{tabular}

Taylor series approach to practical multivariate ruin probability approximations. The improvement is achieved via a substantial reduction in the number of multiple integrals involved in the calculations. A simple numerical illustration of this fact is presented in Section 3. The proof of Theorem 2 is developed in Section 4, and Section 5 is devoted to concluding comments.

\section{An alternative recursive formula}

Let us introduce the following theorem, the proof of which is presented in Section 4.

Theorem 2. In the conditions stated for Theorem 1,

$$
q_{t, u, x, y}^{(i, k)}=\sum_{j=0}^{i}(-1)^{j} \frac{t^{j}}{j !} p_{t, u, x, y}^{(i-j, k)}, \quad i=0,1,2, \ldots \quad k=1,2, \ldots,
$$

where

$$
\begin{aligned}
& p_{t, u, x, y}^{(0, k)}=F_{x, y} * G^{*(k-1)}(u+t) \\
& p_{t, u, x, y}^{(i, k)}=\int_{0}^{t} \int_{0}^{u+s} p_{t-s, u+s-z, x, y}^{(i-1, k)} b(z) \mathrm{d} z \mathrm{~d} s=\mathcal{H}_{1}\left(p_{t, u, x, y}^{(i-1, k)}\right), \quad i=1,2, \ldots \quad k=1,2, \ldots
\end{aligned}
$$

Using (2.2), it is clear that for a fi ed $k$,

$$
\begin{aligned}
& p_{t, u, x, y}^{(1, k)}=\mathcal{H}_{1}\left(p_{t, u, x, y}^{(0, k)}\right), \\
& p_{t, u, x, y}^{(2, k)}=\mathcal{H}_{1}\left(\mathcal{H}_{1}\left(p_{t, u, x, y}^{(0, k)}\right)\right), \\
& p_{t, u, x, y}^{(3, k)}=\mathcal{H}_{1}\left(\mathcal{H}_{1}\left(\mathcal{H}_{1},\left(p_{t, u, x, y}^{(0, k)}\right)\right)\right)
\end{aligned}
$$

It is not hard to conclude, using (2.1), that the number of multiple integrals with known integrands, $q_{t, u, x, y}^{(j, k)}$ and $b(z)$, to evaluate for each $q_{t, u, x, y}^{(j, k)}$ will be only $j$. As a consequence, in this case, for the $i$ th term of the Taylor series expansion, $\Psi_{t, u, x, y}^{(i)}(0) / i$ ! , the total number of integrals is just 
Table 2

\begin{tabular}{|c|c|c|c|c|}
\hline$\lambda$ & $\lambda^{2}$ & $\lambda^{3}$ & $\lambda^{4}$ & $\cdots$ \\
\hline$\Psi_{u, x, y}^{(1)}(0) / 1 !$ & $\Psi_{u, x, y}^{(2)}(0) / 2 !$ & $\Psi_{u, x, y}^{(3)}(0) / 3 !$ & $\Psi_{u, x, y}^{(4)}(0) / 4 !$ & $\ldots$ \\
\hline$-q_{t, u, x, y}^{(0,1)}$ & $\begin{array}{l}-q_{t, u, x, y}^{(0,2)} \\
-q_{t, u, x, y}^{(1,1)}\end{array}$ & $\begin{array}{l}-q_{t, u, x, y}^{(0,3)} \\
-q_{t, u, x, y}^{(1,2)} \\
-q_{t, u, x, y}^{(2,, 1)}\end{array}$ & $\begin{array}{l}-q_{t, u, x, y}^{(0,4)} \\
-q_{t, u, x, y}^{(1,3)} \\
-q_{t, u, x, y}^{(2,2)} \\
-q_{t, u, x, y}^{(3,1)}\end{array}$ & $\begin{array}{l}\cdots \\
\cdots \\
\cdots \\
\cdots\end{array}$ \\
\hline
\end{tabular}

$$
\sum_{j=0}^{i} j=\frac{i(i-1)}{2}
$$

(it can be proved using formula (1.3)).

Moreover, it is important to mention that for the Taylor expansion of order $n$, the total number of integrals is exactly the same as for the $n$th term (2.3) because functions $p_{t, u, x, y}^{(j, k)}$ will be recombined using (2.1) in order to obtain functions $q_{t, u, x, y}^{(j, k)}$.

In Table 1, the total number of multiple integrals to evaluate using both recursive schemes, Theorems 1 and 2 , are displayed for different values of the order of the Taylor expansion.

It is clear that the recursive scheme stated in Theorem 2 reduces significantl the number of multiple integrals to evaluate when calculating the Taylor expansions of order $n$.

\section{Numerical illustration}

Let us illustrate the advantages presented in this paper using a very simple case. We will use Example 1 of our main reference, the paper by Frey and Schmidt (1996), where claim sizes are assumed to be exponential, $1-B(x)=\mathrm{e}^{-\mu x}$. A closed formula can be found for the integrals involved in this illustration.

Let us now obtain the firs three members of the Taylor series expansion. From formula (1.3) or Table 2 it is clear that the following functions must be considered: $q_{t, u, \infty, y}^{(0,1)}, q_{t, u, \infty, y}^{(0,2)}, q_{t, u, \infty, y}^{(0,3)}, q_{t, u, \infty, y}^{(1,1)}, q_{t, u, \infty, y}^{(1,2)}$ and $q_{t, u, \infty, y}^{(2,1)}$.

Using either Theorem 1 or Theorem 2 , the following initial functions must be used:

$$
q_{t, u, \infty, y}^{(0, k)}=p_{t, u, \infty, y}^{(0, k)}=\frac{M(\mu, t, u, y)(u+t)^{k-1}}{(k-1) !}, \quad k=1,2,3
$$

where

$$
M(\mu, t, u, y)=\frac{\left(1-\mathrm{e}^{-\mu y}\right) \mathrm{e}^{-\mu(u+t)}}{\mu} .
$$

Considering the recursive scheme presented in Theorem 1, the functions involved in the calculation of the firs three terms of the Taylor expansion will be expressed using the formulas:

$$
\begin{aligned}
& q_{t, u, \infty, y}^{(1,1)}=\mathcal{H}_{1}\left(q_{t, u, x, y}^{(0,1)}\right)-\mathcal{H}_{2}\left(q_{t, u, x, y}^{(0,1)}\right) \\
& q_{t, u, \infty, y}^{(1,2)}=\mathcal{H}_{1}\left(q_{t, u, x, y}^{(0,2)}\right)-\mathcal{H}_{2}\left(q_{t, u, x, y}^{(0,2)}\right) \\
& q_{t, u, \infty, y}^{(2,1)}=\mathcal{H}_{1}\left(\mathcal{H}_{1}\left(q_{t, u, x, y}^{(0,1)}\right)\right)-\mathcal{H}_{1}\left(\mathcal{H}_{2}\left(q_{t, u, x, y}^{(0,1)}\right)\right)+\mathcal{H}_{2}\left(\mathcal{H}_{1}\left(q_{t, u, x, y}^{(0,1)}\right)\right)-\mathcal{H}_{2}\left(\mathcal{H}_{2}\left(q_{t, u, x, y}^{(0,1)}\right)\right)
\end{aligned}
$$


and the eight integrals (see Table 1) involved are:

$$
\begin{aligned}
& \mathcal{H}_{1}\left(q_{t, u, x, y}^{(0,1)}\right)=\frac{1}{2} \mu t(2 u+t) M(\mu, t, u, y), \\
& \mathcal{H}_{2}\left(q_{t, u, x, y}^{(0,1)}\right)=M(\mu, t, u, y) t, \\
& \mathcal{H}_{1}\left(q_{t, u, x, y}^{(0,2)}\right)=\frac{1}{6} \mu t\left(3 u^{2}+2 t^{2}+6 t u\right) M(\mu, t, u, y), \\
& \mathcal{H}_{2}\left(q_{t, u, x, y}^{(0,2)}\right)=M(\mu, t, u, y) t(u+t), \\
& \mathcal{H}_{1}\left(\mathcal{H}_{1}\left(q_{t, u, x, y}^{(0,1)}\right)\right)=\frac{1}{12} \mu^{2} t^{2}\left(3 u^{2}+4 t u+t^{2}\right) M(\mu, t, u, y), \\
& \mathcal{H}_{1}\left(\mathcal{H}_{2}\left(q_{t, u, x, y}^{(0,1)}\right)\right)=\frac{1}{6} \mu t^{2}(3 u+t) M(\mu, t, u, y), \\
& \mathcal{H}_{2}\left(\mathcal{H}_{1}\left(q_{t, u, x, y}^{(0,1)}\right)\right)=\frac{1}{6} \mu t^{2}(2 t+3 u) M(\mu, t, u, y), \\
& \mathcal{H}_{2}\left(\mathcal{H}_{2}\left(q_{t, u, x, y}^{(0,1)}\right)\right)=\frac{1}{2} t^{2} M(\mu, t, u, y) .
\end{aligned}
$$

Functions $q_{t, u, \infty, y}^{(1,1)}, q_{t, u, \infty, y}^{(1,2)}$ and $q_{t, u, \infty, y}^{(2,1)}$ can also be obtained using the recursion formula (2.2) of Theorem 2 . In this last case, only the following three integrals (see Table 1) will be used:

$$
\begin{aligned}
& p_{t, u, \infty, y}^{(1,1)}=\mathcal{H}_{1}\left(p_{t, u, x, y}^{(0,1)}\right)=\frac{1}{2} \mu t(2 u+t) M(\mu, t, u, y), \\
& p_{t, u, \infty, y}^{(1,2)}=\mathcal{H}_{1}\left(p_{t, u, x, y}^{(0,2)}\right)=\frac{1}{6} \mu t\left(3 u^{2}+2 t^{2}+6 t u\right) M(\mu, t, u, y), \\
& p_{t, u, \infty, y}^{(2,1)}=\mathcal{H}_{1}\left(\mathcal{H}_{1}\left(p_{t, u, x, y}^{(0,1)}\right)\right)=\frac{1}{12} \mu t^{2}\left(3 \mu u^{2}+4 \mu t u-6 u-2 t+\mu t^{2}\right) M(\mu, t, u, y),
\end{aligned}
$$

and combined using (2.1).

In this illustration, a Taylor series expansion of low order, $n=3$, has been considered for the sake of simplicity. Nevertheless, it is enough to show how the number of calculations can be substantially reduced using the recursive scheme presented in Theorem 2.

We should also bear in mind that in this easy example closed formulas can be obtained for the integrals involved in the calculations. When analytical solutions are impossible to obtain and numerical multiple integration is necessary, the mentioned reduction in the number of multiple integrals is most important when controlling the error of the approximations and the computation time.

\section{Proof of Theorem 2}

It is clear from (1.3) that the $n$th term of the infinit sum (1.2), $\left(\Psi_{t, u, x, y}^{(n)}(0) / n !\right) \lambda^{n}$, is obtained from the $n$th column of the following table, multiplying the firs row by the sum of the rest of the members of that column.

We can then defin

$$
\operatorname{column}[j]_{t, u, x, y}(\lambda)=\lambda^{j}\left(\frac{\Psi_{u, x, y}^{(j)}(0)}{j !}-\sum_{k=1}^{j} q_{t, u, x, y}^{(j-k, k)}\right) .
$$


If we want to obtain the series (1.2) we need to use the whole information contained in the infinit number of columns, proceeding as stated above for each and every column and summing up the results;

$$
\Psi_{t, u, x, y}(\lambda)=\sum_{j=1}^{\infty} \operatorname{column}[j]_{t, u, x, y}(\lambda)=\sum_{j=1}^{\infty} \lambda^{j}\left(\frac{\Psi_{u, x, y}^{(j)}(0)}{j !}-\sum_{k=1}^{j} q_{t, u, x, y}^{(j-k, k)}\right)=\sum_{n=1}^{\infty} \frac{\Psi_{t, u, x, y}^{(n)}(0)}{n !} \lambda^{n}
$$

Let us now sum along the different rows of Table 2 instead of using the columns; we can then defin this family of functions:

$$
\begin{aligned}
& \operatorname{row}[1]_{t, u, x, y}(\lambda)=\sum_{k=1}^{\infty} \frac{\Psi_{u, x, y}^{(n)}(0)}{k !} \lambda^{k}=\Psi_{u, x, y}(\lambda), \\
& \operatorname{row}[2]_{t, u, x, y}(\lambda)=-\sum_{k=1}^{\infty} q_{t, u, x, y}^{(0, n)} \lambda^{k}=-\sum_{k=1}^{\infty} \frac{\Psi_{u+t, x, y}^{(n)}(0)}{k !} \lambda^{k}=-\Psi_{u+t, x, y}(\lambda), \\
& \operatorname{row}[j]_{t, u, x, y}(\lambda)=-\lambda^{j-2} \sum_{k=1}^{\infty} q_{t, u, x, y}^{(j-2, k)} \lambda^{k}, \quad j=3,4, \ldots,
\end{aligned}
$$

and as in the case of columns;

$$
\Psi_{t, u, x, y}(\lambda)=\sum_{k=1}^{\infty} \operatorname{row}[k]_{t, u, x, y}(\lambda)
$$

The following lemma will reveal the recursive nature of the family of functions $\operatorname{row}[j]_{t, u, x, y}(\lambda), j=3,4, \ldots$

Lemma 3. The family of functions row $[j]_{t, u, x, y}(\lambda), j=3,4$, ... can be define recursively using integral operators $\mathcal{H}_{1}$ and $\mathcal{H}_{2}(\operatorname{see}(1.6))$ :

$$
\operatorname{row}[j]_{t, u, x, y}(\lambda)=\lambda \mathcal{H}_{1}\left(\operatorname{row}[j-1]_{t, u, x, y}(\lambda)\right)-\lambda \mathcal{H}_{2}\left(\operatorname{row}[j-1]_{t, u, x, y}(\lambda)\right)
$$

Proof. Using (1.4) and (4.1) and trivial properties of the integral operators $\mathcal{H}_{1}$ and $\mathcal{H}_{2}$, for $j=3,4, \ldots$

$$
\begin{aligned}
\operatorname{row}[j]_{t, u, x, y}(\lambda) & =-\lambda^{j-2} \sum_{k=1}^{\infty} q_{t, u, x, y}^{(j-2, k)} \lambda^{k}=-\lambda^{(j-2)} \sum_{k=1}^{\infty}\left[\mathcal{H}_{1}\left(q_{t, u, x, y}^{((j-2)-1, k)}\right)-\mathcal{H}_{2}\left(q_{t, u, x, y}^{((j-2)-1, k)}\right)\right] \lambda^{k} \\
& =\lambda \mathcal{H}_{1}\left(-\lambda^{((j-1)-2)} \sum_{k=1}^{\infty} q_{t, u, x, y}^{((j-1)-2, k)} \lambda^{k}\right)-\lambda \mathcal{H}_{2}\left(-\lambda^{((j-1)-2)} \sum_{k=1}^{\infty} q_{t, u, x, y}^{((j-1)-2, k)} \lambda^{k}\right) \\
& =\lambda \mathcal{H}_{1}\left(\operatorname{row}[j-1]_{t, u, x, y}(\lambda)\right)-\lambda \mathcal{H}_{2}\left(\operatorname{row}[j-1]_{t, u, x, y}(\lambda)\right)
\end{aligned}
$$

Let us now introduce the recursive functions:

$$
\begin{aligned}
& C_{t, u, x, y}^{2}(\lambda)=\Psi_{u+t, x, y}(\lambda), \\
& C_{t, u, x, y}^{j}(\lambda)=\mathcal{H}_{1}\left(C_{t, u, x, y}^{j-1}(\lambda)\right), \quad j=3,4, \ldots
\end{aligned}
$$

and express the family $\operatorname{row}[j]_{t, u, x, y}(\lambda), j=3,4, \ldots$, in terms of the former recursive functions with the next lemma. It is important to notice that the recursive scheme used in (4.3) is simpler than the one proved in Lemma 3 (4.2) 
because only the firs integral operator $\mathcal{H}_{1}$ is involved. Due to this last fact, the proof of Theorem 2 will mainly rely upon the following lemma.

Lemma 4. For $i=2,3, \ldots$ the following expansion holds:

$$
-\operatorname{row}[i]_{t, u, x, y}(\lambda)=\lambda^{i-2} \sum_{j=0}^{i-2}(-1)^{j} \frac{t^{j}}{j !} C_{t, u, x, y}^{i-j}(\lambda)
$$

Proof. Let us proceed by complete induction.

Using (4.2), Lemma 3, it is easy to prove that

$$
\begin{aligned}
& -\operatorname{row}[2]_{t, u, x, y}(\lambda)=\Psi_{u+t, x, y}(\lambda)=C_{t, u, x, y}^{2}(\lambda), \\
& -\operatorname{row}[3]_{t, u, x, y}(\lambda)=\lambda \mathcal{H}_{1}\left(\Psi_{u+t, x, y}(\lambda)\right)-\lambda \mathcal{H}_{2}\left(\Psi_{u+t, x, y}(\lambda)\right)=\lambda C_{t, u, x, y}^{3}(\lambda)-(\lambda t) C_{t, u, x, y}^{2}(\lambda) .
\end{aligned}
$$

Let us suppose now that (4.4) is true for $-\operatorname{row}[i]_{t, u, x, y}$, then using again (4.2),

$$
\begin{aligned}
&-\operatorname{row}[i+1]_{t, u, x, y}(\lambda)=-\lambda\left(\mathcal{H}_{1}\left(\operatorname{row}[i]_{t, u, x, y}(\lambda)\right)-\mathcal{H}_{2}\left(\operatorname{row}[i]_{t, u, x, y}(\lambda)\right)\right) \\
&=\lambda \mathcal{H}_{1}\left(\sum_{j=0}^{i-2} \lambda^{i-2} \frac{(-1)^{j} t^{j}}{j !} C_{t, u, x, y}^{i-j}(\lambda)\right)-\lambda \mathcal{H}_{2}\left(\sum_{j=0}^{i-2} \lambda^{i-2} \frac{(-1)^{j} t^{j}}{j !} C_{t, u, x, y}^{i-j}(\lambda)\right) \\
&=\lambda^{(i+1)-2} C_{t, u, x, y}^{(i+1)}(\lambda)+\lambda^{(i+1)-2} \sum_{j=1}^{i-2}\left(\mathcal{H}_{1}\left(\frac{(-1)^{j} t^{j}}{j !} C_{t, u, x, y}^{i-j}(\lambda)\right)-\mathcal{H}_{2}\left(\frac{(-1)^{j-1} t^{j-1}}{(j-1) !} C_{t, u, x, y}^{i-(j-1)}(\lambda)\right)\right) \\
&+\lambda^{(i+1)-2}(-1)^{(i+1)-2} C_{t, u, x, y}^{2}(\lambda)=\lambda^{(i+1)-2} \sum_{j=0}^{(i+1)-2} \frac{(-1)^{j} t^{j}}{j !} C_{t, u, x, y}^{(i+1)-j}(\lambda)
\end{aligned}
$$

applying the result obtained in Lemma 5 (4.7) to the sum of expression (4.6).

The cited lemma in the former proof designed to simplify (4.6) is now presented and proved.

Lemma 5. For $(i-j)>2$ the following relation holds:

$$
\frac{t^{j+1}}{(j+1) !} C_{t, u, x, y}^{i-j}(\lambda)=\mathcal{H}_{1}\left(\frac{t^{j+1}}{(j+1) !} C_{t, u, x, y}^{i-(j+1)}(\lambda)\right)+\mathcal{H}_{2}\left(\frac{t^{j}}{j !} C_{t, u, x, y}^{i-j}(\lambda)\right) .
$$

Proof. From the definitio of operators $\mathcal{H}_{1}$ and $\mathcal{H}_{2}(1.6)$, it is clear that

$$
\mathcal{H}_{1}\left(\frac{t^{j+1}}{(j+1) !} C_{t, u, x, y}^{i-(j+1)}(\lambda)\right)=\frac{1}{(j+1) !} \int_{0}^{t}(t-s)^{j+1} \int_{0}^{u+s} C_{t-s, u+s-z, x, y}^{i-(j+1)}(\lambda) b(z) \mathrm{d} z \mathrm{~d} s
$$

and

$$
\mathcal{H}_{2}\left(\frac{t^{j}}{j !} C_{t, u, x, y}^{i-j}(\lambda)\right)=\frac{1}{j !} \int_{0}^{t}(t-s)^{j} C_{t-s, u+s, x, y}^{i-j}(\lambda) \mathrm{d} s .
$$

Then 


$$
\begin{aligned}
& \mathcal{H}_{1}\left(\frac{t^{j+1}}{(j+1) !} C_{t, u, x, y}^{i-(j+1)}(\lambda)\right)+\mathcal{H}_{2}\left(\frac{t^{j}}{j !} C_{t, u, x, y}^{i-j}(\lambda)\right) \\
& =\sum_{l=0}^{j+1} \frac{(-1)^{l} t^{(j+1)-l}}{((j+1)-l) ! l !} \mathcal{H}_{1}\left(s^{l} C_{t, u, x, y}^{i-(j+1)}(\lambda)\right)+\sum_{l=0}^{j} \frac{(-1)^{l} t^{j-l}}{(j-l) ! l !} \mathcal{H}_{2}\left(s^{l} C_{t, u, x, y}^{i-j}(\lambda)\right) \\
& =\frac{t^{j+1}}{(j+1) !} C_{t, u, x, y}^{i-j}(\lambda)+\sum_{l=0}^{j} \frac{(-1)^{l} t^{j-l}}{(j-l) ! l !}\left(\frac{(-1)}{l+1} \mathcal{H}_{1}\left(s^{l+1} C_{t, u, x, y}^{i-(j+1)}(\lambda)\right)+\mathcal{H}_{2}\left(s^{l} C_{t, u, x, y}^{i-j}(\lambda)\right)\right) \\
& =\frac{t^{j+1}}{(j+1) !} C_{t, u, x, y}^{i-j}(\lambda)
\end{aligned}
$$

because,

$$
\begin{aligned}
\mathcal{H}_{2}\left(s^{l} C_{t, u, x, y}^{i-j}(\lambda)\right) & =\int_{0}^{t} s^{l} C_{t-s, u+s, x, y}^{i-j}(\lambda) \mathrm{d} s=\int_{s=0}^{t} s^{l} \int_{m=0}^{t-s} \int_{z=0}^{u+s+m} C_{t-s-m, u+s+m-z, x, y}^{i-j-1}(\lambda) b(z) \mathrm{d} z \mathrm{~d} m \mathrm{~d} s \\
& =\left(\frac{1}{l+1}\right) \int_{\varkappa=0}^{t} \varkappa^{l+1} \int_{z=0}^{u+\varkappa} C_{t-\varkappa, u+\varkappa-z, x, y}^{i-j-1}(\lambda) b(z) \mathrm{d} z \mathrm{~d} \varkappa=\frac{1}{l+1} \mathcal{H}_{1}\left(s^{l} C_{t, u, x, y}^{i-(j+1)}(\lambda)\right)
\end{aligned}
$$

using the following change of variables;

$$
m+s=\varkappa, \quad s=s, \quad z=z .
$$

and shifting the limits of the firs two integrals.

Let us now defin the following family of functions (see (2.2)):

$$
p_{t, u, x, y}^{(0, k)}=F_{x, y} * G^{(k-1)}(u+t), \quad p_{t, u, x, y}^{(i, k)}=\mathcal{H}_{1}\left(p_{t, u, x, y}^{(i-1, k)}\right), \quad i=1,2, \ldots \quad k=1,2, \ldots
$$

As it was stated in Theorem 1 of the original paper by Frey and Schmidt (1996):

$$
C_{t, u, x, y}^{2}(\lambda)=\Psi_{u+t, x, y}(\lambda)=\sum_{k=1}^{\infty} \frac{\Psi_{u+t, x, y}^{(n)}(0)}{k !} \lambda^{k}=\sum_{k=1}^{\infty} F_{x, y} * G^{(k-1)}(u+t) \lambda^{k}=\sum_{k=1}^{\infty} p_{t, u, x, y}^{(0, k)} \lambda^{k},
$$

then using the recursive formulas (4.2) and (2.2) it is easy to prove that

$$
C_{t, u, x, y}^{n}(\lambda)=\sum_{k=1}^{\infty} p_{t, u, x, y}^{(n-2, k)} \lambda^{k}, \quad n=2,3,4, \ldots
$$

Using the result of Lemma 4 (4.4) along with the former expansion (4.8)

$$
\begin{aligned}
-\operatorname{row}[i] & =\lambda^{i-2} \sum_{j=0}^{i-2}(-1)^{j} \frac{t^{j}}{j !} C_{t, u, x, y}^{i-j}(\lambda)=\lambda^{i-2} \sum_{j=0}^{i-2}(-1)^{j} \frac{t^{j}}{j !} \sum_{k=1}^{\infty} \lambda^{k} p_{t, u, x, y}^{(i-j-2, k)} \\
& =\lambda^{i-2} \sum_{k=1}^{\infty} \lambda^{k}\left(\sum_{j=0}^{i-2}(-1)^{j} \frac{t^{j}}{j !} p_{t, u, x, y}^{(i-j-2, k)}\right)=\lambda^{i-2} \sum_{k=1}^{\infty} \lambda^{k} q_{t, u, x, y}^{(i-2, k)} \quad i=2,3, \ldots
\end{aligned}
$$

subsequently,

$$
q_{t, u, x, y}^{(i-2, k)}=\sum_{j=0}^{i-2}(-1)^{j} \frac{t^{j}}{j !} p_{t, u, x, y}^{(i-j-2, k)}, \quad i=2,3, . .
$$


and finall ,

$$
q_{t, u, x, y}^{(i, k)}=\sum_{j=0}^{i}(-1)^{j} \frac{t^{j}}{j !} p_{t, u, x, y}^{(i-j, k)}, \quad i=0,1, . .
$$

\section{Concluding comments}

The Taylor series expansion Eq. (1.2) with respect the arrival intensity, $\lambda$, of the multivariate ruin probabilities was firs introduced, to our knowledge, in risk theory by Frey and Schmidt (1996). This new approach can be considered as an original and alternative approach in approximating multivariate characteristics of classical ruin processes other than inversion of Laplace transforms for particular claim size distributions (Gerber et al., 1987; Dufresne and Gerber, 1988a,b) or discretization of the claim size and time (Dickson, 1989, 1993; Dickson and Waters, 1992) applying the so-called Panjer's recursive algorithm (Panjer, 1981)).

In the original work by Frey and Schmidt (1996), the terms of the Taylor series expansion with respect to the arrival intensity, $\lambda$, of the multivariate ruin probabilities, were obtained recursively using Theorem 1 , expressions (1.3) and (1.4). The number of multiple integrals involved in the calculations increases exponentially with the order of the Taylor expansions considered (see Table 1, second column). This last fact clearly affects the implementation possibilities of this interesting approach.

For the reason just mentioned above, we showed with Theorem 2, expressions (2.1) and (2.2), that a simplificatio in the recursive scheme can enhance the implementation of the Taylor series approach to practical multivariate ruin probability approximations. The improvement is achieved via a substantial reduction in the number of multiple integrals involved in the calculations (see Table 1).

The reduction in the number of multiple integrals becomes most important when analytical solutions are impossible to obtain and numerical multiple integration is necessary. In this context, computation times are, obviously, proportional to the number of multiple integrals to evaluate.

Nevertheless, the applicability matters of this interesting approach, Taylor series expansion, to multivariate finit time ruin probabilities is certainly an appealing area of future study by academic actuaries. For instance, the application of Laplace transforms to the recursive schemes based on the results contained in Rolski et al. (1999), Sections 5.5 and 8.3.2, or the study of numerical methods suitable to solve the multiple integrals generated in the recursive schemes (see, for instance, Usábel (1998)).

The following corollary of Theorem 2 could be useful for future works on this topic. In this last result, the recursive scheme used to obtain the derivatives of the finit time multivariate ruin probability is presented in a more straightforward fashion.

\section{Corollary 6.}

$$
\frac{\Psi_{t, u, x, y}^{(n)}(0)}{n !}=\frac{\Psi_{u, x, y}^{(n)}(0)}{n !}-\left(\sum_{l=0}^{n-1}(-1) \frac{l^{l}}{l !} \mathcal{F}_{t, u, x, y}^{n-l}\right),
$$

where

$$
\begin{aligned}
& \mathcal{F}_{t, u, x, y}^{1}=F_{x, y}(u+t) \\
& \mathcal{F}_{t, u, x, y}^{k}=\int_{0}^{t} \int_{0}^{u+s} \mathcal{F}_{t-s, u+s-z, x, y}^{k-1} b(z) \mathrm{d} z \mathrm{~d} s+F_{x, y} * G^{(k-1)}(u+t), \quad k=2,3, \ldots
\end{aligned}
$$

Proof. Using (2.1) and the result of Theorem 1 


$$
\frac{\Psi_{t, u, x, y}^{(n)}(0)}{n !}=\frac{\Psi_{u, x, y}^{(n)}(0)}{n !}-\left(\sum_{l=0}^{n-1}(-1)^{l} \frac{t^{l}}{l !}\left(\sum_{i=1}^{n-l} p_{t, u, x, y}^{(n-l-i, i)}\right)\right)
$$

definin

$$
\mathcal{F}_{t, u, x, y}^{k}=\sum_{i=1}^{k} p_{t, u, x, y}^{(k-i, i)}, \quad k=1,2, \ldots
$$

and substituting we finall get the statement of the corollary.

$$
\frac{\Psi_{t, u, x, y}^{(n)}(0)}{n !}=\frac{\Psi_{u, x, y}^{(n)}(0)}{n !}-\left(\sum_{l=0}^{n-1}(-1)^{l} \frac{t^{l}}{l !} \mathcal{F}_{t, u, x, y}^{n-l}\right) \text {. }
$$

\section{Acknowledgements}

The author gratefully acknowledges the financia support from Programa Sectorial de Becas de Formacion de Profesorado y Perfeccionamiento de Personal Investigador, Ministerio de Educacion y Cultura, España (1999).

\section{References}

Dickson, C., 1989. Recursive calculation of the probability and severity of ruin. Insurance: Mathematics and Economics 8, 145-148.

Dickson, C., Waters, H., 1992. The probability and severity of ruin in finit and infinit time. ASTIN Bulletin 22 (2), 177-190.

Dickson, C., 1993. On the distribution of the claim causing ruin. Insurance: Mathematics and Economics 12, 143-154.

Dufresne, F., Gerber, H., 1988a. The probability and severity of ruin for combinations of exponential claim amount distributions and their translations. Insurance: Mathematics and Economics 7, 75-80.

Dufresne, F., Gerber, H., 1988b. The surpluses immediately before and at ruin, and the amount of the claim causing ruin. Insurance: Mathematics and Economics 7, 193-199.

Frey, A., Schmidt, V., 1996. Taylor Series expansion for multivariate characteristics of classical risk processes. Insurance: Mathematics and Economics 18, 1-12.

Gerber, H., Goovaerts, M., Kaas, R., 1987. On the probability and severity of ruin. ASTIN Bulletin 17 (2), 151-163.

Panjer, H.H., 1981. Recursive calculation of a family of compound distributions. ASTIN Bulletin 12, 22-26.

Rolski, T., Schmidli, H., Schmidt, V., Teugels, J., 1999. Stochastic Processes for Insurance and Finance. Wiley, Chichester.

Usábel, M.A., 1998. Applications to Risk Theory of a Monte Carlo multiple integration method. Insurance: Mathematics and Economics 23 (1), 71-84. 